\title{
O QUE ALUNOS DE DIFERENTES CURSOS PROCURAM EM DISCIPLINAS EXTRACURRICULARES DE BIOQUÍMICA?
}

\author{
Daniela K. Yokaichiya ${ }^{1 *}$, Eduardo Galembeck ${ }^{1}$, Bayardo Baptista Torres ${ }^{2}$ \\ ${ }_{1}^{1}$ Laboratório de Tecnologia Educacional - Depto. de Bioquímica - IB - Unicamp \\ ${ }^{2}$ Laboratório de Educação em Bioquímica - Depto. de Bioquímica - IQ - USP \\ *dycy@unicamp.br
}

\begin{abstract}
Resumo:
Ao mesmo tempo que a seleção detalhada dos conteúdos direciona as aulas de Bioquímica aos interesses de cada curso de graduação, muitos assuntos são inevitavelmente excluídos. A procura por disciplinas não obrigatórias no currículo mínimo que abordem temas de Bioquímica não contemplados nas disciplinas de Bioquímica Básica aponta para o grande interesse dos estudantes por complementação na sua formação [1]. Na ocasião do oferecimento de um curso sobre Bioquímica da Nutrição a distância em 2001 e 2003, foi feito um levantamento de interesse pelo curso, além de uma contínua avaliação da satisfação dos alunos frente às atividades desenvolvidas. A análise detalhada dos interesses e das críticas e sugestões ajudou a verificar os anseios dos diferentes profissionais em relação aos conteúdos da Bioquímica e suas aplicações. Dessa análise conclui-se que apesar dos alunos entrarem com interesse específico de aprofundamento nos conteúdos de suas áreas, o que promove maior satisfação é a interação e troca de informações - ainda que relativamente superficiais - com alunos e profissionais de outras formações. Cursos que visem aprofundamento específico de determinados assuntos devem, entretanto, ser oferecidos para públicos mais restritos, atendendo a pré-requisitos de conhecimento para que as discussões possam ser mais aprofundadas.
\end{abstract}

\begin{abstract}
:
The rigorous selection of contents to be included in a Biochemistry course both attends the interest of each specific undergraduation course and excludes many subjects of general interest. The great number of enrollment in non-obligatory courses dealing with subjects not deeply treated in most introductory Biochemistry course suggests the concern of the students in improving their knowledge in the field [1]. During the Biochemistry of Nutrition distance education course in 2000 and 2003, we collected studentsreports about their interest on the course and their satisfaction with the developed activities. For this purpose we made several courses evaluation (during and at the end of each course). The detailed analysis of these data let us to verify studentsexpectations related to the Biochemistry contents according to their graduate courses. From this analysis, it was possible to conclude that even though students from different courses have specific interests in biochemistry contents, the aspect that promoted more satisfaction in Biochemistry of Nutrition distance education course was the participantsinteraction and the exchange of information even superficially considered among students with different knowledge background. Nevertheless, courses about specific contents should be offered to a more restrict public, with restrictive pre-requisite so that deepening discussions can be held
\end{abstract}




\section{Introdução}

A Bioquímica sempre foi considerada uma disciplina difícil de ser ministrada pela complexidade de seus conteúdos, visto que trata de fenômenos micro e macromoleculares, difíceis de serem abstraídos e compreendidos. Outra dificuldade do ensino da Bioquímica diz respeito ao grande volume de informações atualmente disponíveis e que devem ser restringidas para acomodar-se ao tempo disponível nas disciplinas de Bioquímica Básica. Muitas iniciativas têm sido desenvolvidas para tornar o ensino de Bioquímica mais atraente e interessante para diferentes públicos, tais como alunos de Educação Física [2-5], Medicina [6], Nutrição [6;7], Farmácia e Bioquímica [8], entre outros. Essas iniciativas procuram abordar a Bioquímica vinculando-a, desde o início ao cotidiano e aos interesses dos diferentes profissionais. Todas apresentam resultados satisfatórios tanto para o ensino quanto para o aprendizado.

O rigor da seleção de conteúdos que permite direcionar o currículo de Bioquímica aos interesses de cada curso de graduação impõe a exclusão de muitos assuntos de interesse. A procura por cursos eletivos (não obrigatórios no currículo mínimo) que abordem assuntos de Bioquímica não contemplados nas disciplinas de Bioquímica Básica aponta para o grande interesse por complementação de sua formação por profissionais e futuros profissionais de formações diversas [1]. Dentre os cursos já estruturados para este fim [9-13], está o curso a distância Bioquímica da Nutrição [14], objeto de estudo deste trabalho. Na ocasião do oferecimento da Bioquímica da Nutrição a distância em 2001 e 2003, foi feito um levantamento de interesse dos alunos participantes pelo curso, além de uma contínua avaliação da satisfação dos alunos frente às atividades desenvolvidas.

A análise detalhada dos interesses e das críticas e sugestões manifestadas pelos alunos que participaram das duas experiências do curso a distância "Bioquímica da Nutrição" possibilita verificar os anseios dos diferentes profissionais em relação aos conteúdos da Bioquímica e suas aplicações.

\section{Material e Métodos}

Os alunos matriculados nos cursos Bioquímica da Nutrição de 2001 e 2003 (total de 113 alunos) preencheram um formulário eletrônico disponível no site do Laboratório de Tecnologia Educacional (LTE - Unicamp), onde deveriam ser apontados os interesses que os levaram a se matricular no curso, entre outras informações.

As declarações foram divididas em cinco categorias de resposta:

1. interesse pelos assuntos tratados e o gosto pela bioquímica;

2. interesse relacionado a perspectivas presentes/futuras de aplicação dos conceitos na área de atuação;

3. interesse por mais conhecimento na área (relacionados à alimentação e saúde, bioquímica, química e fenômenos relacionados ao cotidiano) e/ou por revisar a bioquímica;

4. possibilidade de interação com pessoas de diferentes cursos e formações;

5. interesse em experimentar um curso a distância.

As classificações das declarações dos alunos foram feitas por três analisadores, através de análise de conteúdo. Os analisadores classificaram cada mensagem em uma ou mais categorias (uma vez que não são excludentes), de acordo com a sua interpretação. As classificações discordantes foram discutidas e reavaliadas para que os dados fossem tratados com o mesmo critério por todos os analisadores e para dar confiabilidade às conclusões feitas a partir desta análise. Foram somadas as quantidades de cada categoria por analisador por curso (e.g.: na classificação feita pelo analisador 1, foram contabilizadas quantas ocorrências de cada categoria foram encontradas para as manifestações dos alunos de Química, Biologia, Engenharia de Alimentos, etc). Calculou-se a média (dos três analisadores) das ocorrências de cada categoria por curso e a partir delas, pôde-se determinar as porcentagens relativas das ocorrências de cada categoria de interesse por curso.

Os resultados numéricos foram triangulados com declarações dos alunos sobre diversos aspectos do curso nas avaliações parcial (avaliação formativa) e final (avaliação somativa) aplicadas durante os cursos. 


\section{Resultados e Discussão}

A Bioquímica da Nutrição a Distância foi oferecida pela Universidade de São Paulo (USP), como disciplina optativa de graduação e pela Universidade Estadual de Campinas (Unicamp), como disciplina eletiva de graduação e como curso de extensão, através da sua Escola de Extensão (Extecamp). O oferecimento do curso pela Extecamp permitiu a participação de profissionais da área da saúde, não restringindo o público a alunos de graduação, conforme apresentados na tabela I. Os alunos que participaram das duas disciplinas eram na maioria dos cursos de Ciências Nutricionais $(33,3 \%)$ e Ciências Biológicas (20,1\%). Representando uma parcela significativa da amostragem, houve a participação de alunos e profissionais dos cursos de Educação Física (15,8\%), Farmácia e Bioquímica (12,3\%) e Química (8,8\%). Em menor número, estão representados alunos dos cursos de Medicina, Enfermagem e Engenharia de Alimentos $(9,6 \%)$.

\begin{tabular}{|c|c|c|c|}
\hline cursos & Graduandos & Formados & Total \\
\hline Ciências Nutricionais & 31 & 7 & 38 \\
\hline Ciências Biológicas & 17 & 4 & 22 \\
\hline Farmácia e Bioquímica & 13 & 1 & 14 \\
\hline Educação Física & 12 & 6 & 18 \\
\hline Química & 9 & 1 & 10 \\
\hline Medicina / Enfermagem & 4 & 5 & 9 \\
\hline Engenharia de Alimentos & 2 & - & 2 \\
\hline total & 88 & 25 & 113 \\
\hline
\end{tabular}

As categorias criadas para classificar as manifestações de interesse dos alunos não são excludentes e estão listadas em Métodos. Os resultados são apresentados discriminando os cursos de origem dos alunos.

Tabela II: Quantidades (médias e respectivas porcentagens) de cada categoria (listadas em métodos) de interesse dos alunos matriculados na Bioquímica da Nutrição em 2001 e 2003, por curso.

\begin{tabular}{ccccccccc}
\hline \multicolumn{10}{c}{ Cursos } \\
\hline Categorias & Quim. & Ed.Fis. & Eng.Alim. & Cien.Biol. & Cien.Farm. & Med./Enf. & Nutri. & total \\
$\mathbf{1}$ & 5,3 & 3 & 1,6 & 7,6 & 3,6 & 1,6 & 4,3 & 27,3 \\
& $(19,4 \%)$ & $(11 \%)$ & $(5,8 \%)$ & $(27,8 \%)$ & $(13,2 \%)$ & $(5,8 \%)$ & $(15,7 \%)$ & \\
$\mathbf{2}$ & 1,3 & 9,3 & 1 & 7 & 2 & 5 & 11,3 & 37 \\
& $(3,5 \%)$ & $(25,1 \%)$ & $(2,7 \%)$ & $(18,9 \%)$ & $(5,4 \%)$ & $(13,5 \%)$ & $(30,5 \%)$ & \\
$\mathbf{3}$ & 6 & 14 & 1,6 & 16,6 & 11 & 7 & 33,6 & 89,6 \\
& $(6,7 \%)$ & $(15,6 \%)$ & $(1,8 \%)$ & $(18,5 \%)$ & $(12,3 \%)$ & $(7,8 \%)$ & $(37,5 \%)$ & \\
$\mathbf{4}$ & 1 & 0 & 0 & 0 & 0,3 & 0,3 & 1,6 & 3 \\
& $(33,3 \%)$ & 0 & & 5,3 & $(10 \%)$ & $(10 \%)$ & $(53,3)$ & 3 \\
$\mathbf{5}$ & 0 & 0 & 0 & $(57 \%)$ & $(21,5 \%)$ & 0 & $(21,5 \%)$ & 9,3 \\
* Quim. = Química; Ed.Física = Educação Física; Eng.Alim. = Engenharia de Alimentos; Cien.Biol. = Ciências
\end{tabular}

Biológicas; Cien.Farm. = Ciências Farmacêuticas; Med./Enf. = Medicina e Enfermagem (somados); Nutri. = Nutrição.

Tabela III: Porcentagens relativas de cada categoria (listadas em métodos) de interesse dos alunos matriculados na Bioquímica da Nutrição em 2001 e 2003, por curso.

\begin{tabular}{ccccccccc}
\hline \multicolumn{10}{c}{ Categorias } & Quim. & Ed.Fis. & Eng.Alim. & Cien.Biol. & Cien.Farm. & Med./Enf. & Nutri. & total \\
\hline $\mathbf{1}$ & 53 & 16,7 & 80 & 34,5 & 25,7 & 17,7 & 11,3 & 24,1 \\
$\mathbf{2}$ & 13 & 51,6 & 50 & 31,8 & 14,3 & 55,5 & 29,7 & 32,7 \\
$\mathbf{3}$ & 60 & 77,7 & 80 & 75,5 & 78,6 & 77,7 & 88,4 & 79,3 \\
$\mathbf{4}$ & 10 & 0 & 0 & 0 & 2,1 & 3,3 & 4,2 & 2,6 \\
$\mathbf{5}$ & 0 & 0 & 0 & 24,1 & 14,3 & 0 & 5,2 & 8,2
\end{tabular}

* Quim. = Química; Ed.Física = Educação Física; Eng.Alim. = Engenharia de Alimentos; Cien.Biol. = Ciências Biológicas; Cien.Farm. = Ciências Farmacêuticas; Med./Enf. = Medicina e Enfermagem (somados); Nutri. = Nutrição.

De acordo com resultados apresentados na tabela III, o motivo de interesse para a maioria $(79,3 \%)$ é a aquisição de mais conhecimentos e o interesse por revisão dos conteúdos de 
bioquímica (categoria 3). Este interesse aparece nas mesmas proporções dentro dos cursos pesquisados, com menor ênfase no curso de Química $(60 \%)$ e maior no curso de Nutrição $(88,4 \%)$. Admite-se como aquisição de conhecimento, as manifestações de aprofundamento em assuntos relacionados à nutrição, bioquímica, saúde, química, conforme exemplificado nos excertos abaixo (as manifestações estão transcritas exatamente como os alunos escreveram nos formulários e avaliações):

\section{Excerto 1}

Como no meu estágio costumamos alimentar ratas com diferentes tipos de dietas, seria interessante que eu soubesse mais a respeito de cada tipo de alimento, etc. Tbém gostaria de rever muitos conceitos de bioquímica que já foram esquecidos. (aluno de Biologia)

\section{Excerto 2}

Trabalho com alimentos transgênicos e quero saber mais informações sobre os mesmos.

\section{Excerto 3} (aluno de Química)

Melhorar o meu nível de conhecimento e abrir mais uma frente de atuação dentro da

\section{Excerto 4} bioquímica. (aluno de Farmácia e Bioquímica)

Pretendo me especializar em bioquímica, por enquanto a realização deste curso me proporcionará um aprofundamento na matéria e uma visão mais crítica sobre a nutrição

\section{Excerto 5} em minha área (esportiva) (aluno de Educação Física)

Meu interesse é de recordar os meus conhecimentos de bioquímica, além de atualização de informações referentes a esse assunto. Meu interesse é de aprender e expandir

\section{Excerto 6} conhecimentos. (aluno de Nutrição)

Aprofundar-me no assunto e ser orientado de como prosseguir neste sentido, aprendendo inclusive como fazer pesquisas nesta área. Conhecer mais e melhor profissionais e instituições que lidam profissionalmente com esta especialidade. (aluno de Medicina)

A segunda categoria mais apontada pelos alunos foi a perspectiva futura na área de atuação (categoria $2-32,7 \%$ ), indicando interesse em obter mais conhecimentos que possam ser úteis nas futuras atividades. Comparando a manifestação deste interesse nos diferentes cursos (tabela III), observa-se que alunos e profissionais dos cursos de Medicina/Enfermagem, Educação Física e Engenharia de Alimentos são os que demonstram maior interesse em procurar complementação na formação para aplicar os conhecimentos de bioquímica nas atividades profissionais futuras. É curioso observar a pouca manifestação pelos profissionais do curso de Nutrição $(29,7 \%)$, que apresentaram porcentagens de interesse menores do que alunos e profissionais do curso de Ciências Biológicas (31,8\%). Exemplos das manifestações de interesse pela perspectiva de atuação na área estão apresentados a seguir:

\section{Excerto 7}

Como minha área de atuação envolve atletas profissionais no clube e pacientes de doenças crônicas, que envolvem metabolismo, como diabetes e hipertensão, tenho necessidade de dominar este assunto. Espero adquirir conhecimentos que me ajudem a desenvolver melhor a função de orientador nutricional a meus pacientes, para que eles

\section{Excerto 8} tenham mais qualidade de vida. (aluno de Medicina)

Trabalho numa clínica de personal training e a nutrição está presente todos os dias no meu trabalho, então preciso de um conhecimento ainda maior nessa área. Espero com o curso me aprimorar mais, conhecer mais e usá-lo no meu dia-a-dia da melhor maneira

\section{Excerto 9} possível. (aluno de Educação Física)

Fiz cursos de fundamentos de bioquímica semestre passado e simplesmente adorei! Qdo vi o cartaz, fiquei muito interessada, uma vez que a saúde está extremamente ligada a alimentação, e como pretendo trabalhar nessa área, resolvi me inscrever. (aluno de Engenharia de Alimentos) 
Outro motivo de interesse bastante apontado e que deve ser considerado diz respeito ao gosto pela bioquímica e interesse pelos assuntos tratados no curso (categoria 1). Este interesse foi fortemente manifestado por alunos do curso de Engenharia de Alimentos (80\%) e em menores escalas, por alunos de Química $(53 \%)$ e Ciências Biológicas $(34,5)$.

\section{Excerto 10}

\section{Excerto 11}

Meu interesse é bastante grande, pois gosto muito de bioquímica e me interesso bastante por assuntos envolvendo nutrição. (aluno de Biologia)

Gostaria de aprender mais sobre nutrição, pois acho uma área muito interessantes, e acho que no curso de graduação que faço não terei o enfoque que esta disciplina terá.

\section{Excerto 12} (alunos de Química)

Gostei muito de estudar bioquímica o semestre passado e me interesse muito tbém pos

\section{Excerto 13} assuntos da área de nutrição e saúde. (aluno de Engenharia de Alimentos)

Sempre me interessei muito por bioquímica, com certeza é minha matéria favorita na faculdade (empata com biologia molecular). Tenho muito interesse em assuntos ligados a radicais livres e as variações de alimentação que podem deixar ou não um indivíduo mais

\section{Excerto 14} susceptível (ou não) a uma determinada patologia. (aluno de Farmácia e Bioquímica)

Como gosto de Bioquímica e faço Nutrição, achei este curso muito interessante para me aperfeiçoar nesta área. (aluno de Nutrição)

O fato do curso ser a distância e possibilitar interação com profissionais e alunos de variados cursos também foi apontado como um fator de interesse pelos alunos, ainda que em proporções muito menores comparado às demais categorias. Ainda assim, nas avaliações realizadas nos dois cursos (2001 e 2003), houve grande manifestação de satisfação dos alunos pelo uso de tecnologia e pela interação entre os participantes, conforme ilustram os excertos a seguir:

\section{Excerto 15}

Me interesso bastante por bioquímica e achei a idéia de realizar um curso a distância muito interessante. Além de ser uma experiência nova e imagino ser estimulante sair um pouco da sala de aula e aprender discutindo com os demais alunos do curso. (aluno de

\section{Excerto 16} Farmácia e Bioquímica)

(Tenho interesse de) Passar por essa nova experiência de cursos a distância e aprender alguns conhecimentos específicos, visto que essa disciplina está aprofundando os conhecimentos de nutrição. Além disso, debater com pessoas de outras áreas e universidades será muito proveitoso. (aluno de Nutrição)

Nas avaliações parcial e final do curso a distância Bioquímica da Nutrição, observou-se muitas manifestações de satisfação com os temas trabalhados (excertos 17 a 19), com a possibilidade de revisão e aprofundamento de conteúdos (excerto 20 e 21), com a possibilidade de utilizar os conhecimentos nas áreas de atuação (excertos 22 e 23) e com a oportunidade de participar de um curso a distância (excerto 24 e 25). Apesar de ter sido pouco manifestado o interesse, foi grande a ocorrência de manifestação de satisfação em interagir com pessoas de outras formações (excerto 25 a 28), se não o ponto de satisfação mais destacado nas avaliações:

\section{Excerto 17}

Gostaria de parabenizá-los pelo esforço em tentar melhorar cada vez mais a disciplina. Ela é muito interessante e com tópicos muito bem escolhidos. (aluno de biologia)

\section{Excerto 18}


Toda equipe está realmente de parabéns! A disciplina foi ótima (do ponto de vista de conteúdo e organizacional)... os monitores são extremamente prestativos... resumindo

\section{Excerto 19}

"ÓTIMO". (aluno de Farmácia e Bioquímica)

Eu estou adorando o curso! É melhor do que eu imaginava! o conteúdo é muito interessante, abordou mais assuntos do que o que eu vi na disciplina de bioquímica na faculdade e isso é muito estimulador. (aluno de Nutrição)

\section{Excerto 20}

(...) Estou achando o curso ótimo, muito além das expectativas de uma pobre "professora de Ed. Física". Não só aprendi muito, mas também fiquei altamente motivada a continuar querendo saber mais sobre esse mundo ligado à nutrição. (aluno de Educação Física)

\section{Excerto 21}

Para mim, este curso está sendo bom, pois me proporcionou rever alguns conhecimentos já esquecidos e me atualizar sobre outros. (aluno de Nutrição)

\section{Excerto 22}

O curso foi muito interessante e prazeroso de ser realizado, além de ter trazido

\section{Excerto 23} informações sobre o meu projeto de Doutorado (aluno de Química)

O curso foi de grande valia para a construção de nosso conhecimento específico, através dos estudos e debates. Serviu também para vermos o quanto temos que estudar e buscar crescer sempre nas nossas respectivas atuações profissionais. (aluno de Educação Física)

\section{Excerto 24}

Eu gostei muito dessa disciplina, não só pelo conteúdo, mas principalmente pelo seu formato, aliás, o que mais me motivou a cursar essa disciplina foi o fato de ser a

\section{Excerto 25} distância. (aluno de Educação Física)

(...) aproveitei e estou aproveitando bastante, creio que disciplinas a distância são recursos eficientes para o aprendizado. Possibilitam também a integração de alunos de diferentes cursos apesar de não termos contato rela, só virtual. A troca de idéias faz todos "abrirem a cabeça" mesmo, e aprenderem muita coisa! Vale a pena participar de uma disciplina via net, é tão eficiente quanto assistir às aulas convencionais... (aluno de Nutrição)

\section{Excerto 26}

(...) As salas de discussão são as mais interessantes, pois durante a discussão foram levantadas, várias vezes, questões com pontos de vista variados, pela existência de alunos de diferentes cursos. Acho que foi a melhor forma de conseguirmos fixar a

\section{Excerto 27} matéria. (aluno de Engenharia de Alimentos)

O fato de que alunos (são) de diferentes áreas cursem a matéria é interessante, já que

\section{Excerto 28}

Apesar de estarem me dando muito trabalho, estou adorando o curso. Acho que essa possibilidade de diferentes níveis de formação e de diferentes cursos (interagirem) em um mesmo local muito rico para todos. (aluno de Educação Física)

Os excertos acima refletem a maioria das manifestações dos alunos, mas deve-se considerar a ocorrência de algumas manifestações críticas e opostas às descritas anteriormente. Alguns alunos apontaram para o desconforto em discutir alguns temas com pessoas de diferentes níveis de formação. Houve críticas para a dificuldade de linguagem usada nas diferentes áreas e do nível de aprofundamento (mais superficial ou mais complexo) nas discussões.

\section{Conclusões}


O interesse pela bioquímica e por atualização dos seus conteúdos é bastante evidente nos resultados encontrados. Esse resultado já era esperado uma vez que os entrevistados fazem parte do grupo de alunos que efetivamente procuraram e se matricularam no curso de Bioquímica da Nutrição, mostrando de antemão o gosto pela Bioquímica ou o interesse por complementação na formação acadêmica e profissional.

O grande interesse por parte dos alunos de Química e Engenharia de Alimentos em adquirir mais conhecimento para usar futuramente aponta para a interdisciplinaridade possível desses cursos tipicamente da área de exatas.

Os alunos do curso de Nutrição não foram enfáticos em demonstrar gosto pela Bioquímica e/ou interesse pelos assuntos abordados, mas apontaram para a necessidade de revisão dos conceitos e de aprofundar os conhecimentos com discussões travadas com alunos e profissionais de outras formações. $O$ interesse por troca de idéias é um bom indicativo da necessidade por aplicação efetiva dos conhecimentos no campo de atuação profissional. Cursos baseados em Problemas (Problem Based Learning - PBL) seriam interessantes estratégias de ensino para abordar a Bioquímica dentro dos cursos mais aplicados como a Nutrição, a Medicina e Enfermagem e a Educação Física.

Cursos que possibilitem interação e troca com alunos e profissionais de outras formações seriam extremamente bem vindos em cursos que visem a integração dos participantes e a troca de informações, como foi o caso da Bioquímica da Nutrição. Cursos que visem aprofundamento específico de determinados assuntos devem, entretanto, ser oferecidos para públicos mais restritos, atendendo a pré-requisitos de conhecimento para que as discussões possam ser mais aprofundadas.

\section{Referências Bibliográficas}

[1] Yokaichiya, D.K.; Galembeck, E. and Torres, B.B. (2001) EXPECTATIVAS E FATORES DE INTTERESSSE POR ENSINO A DISTÂNCIA NA ÁREA DE BIOQUÍMICA: RELATOS DE UMA PESQUISA PRÉ E PÓS APLICAÇÃO DE UMA DISCIPLINA DE BIOQUÍMICA A DISTÂNCIA. Revista Brasileira de Ensino de Bioquímica - http://sbbq.iq.usp.br/revista/artigo.php?artigoid=38.

[2] Santos, E.A.S.; Pires, T.C., Andrade, G.P.V., Chavante, S.F., Sales, M.P. and Oliveira, F.W. (2002) TEACHING BIOCHEMISTRY USING AN INTEGRATIVE AND CONTEXTUAL APPROACH. Revista Brasileira de Ensino de Bioquímica e Biologia Molecular - http://www.sbbq.org.br/revista/artigo.php?artigoid=58

[3] Galembeck, E.; Macedo, D.V. and Torres, B.B. (2003) DIFFERENT ENERGY SOURCES IN SPORTS. Bambed, 31(3):204-208.

[4] Macedo, D.V.; Hohl, R.; Lazarim, F. L.; Silva, F. O. C.; Dias, R. G.; Gandra, P. G.; Tessutti, L. S. and Pereira, M. L. (2003) A PRACTICAL APPROACH FOR A BASIC BIOCHEMISTRY COURSE TO PHYSICAL EDUCATION STUDENTS, Revista Brasileira de Ensino de Bioquímica e Biologia Molecular http://www.sbbq.org.br/revista/artigo.php?artigoid=77

[5] Da Costa, C. and Torres, B.B. (2004) ADJUSTING A BIOCHEMISTRY COURSE FOR PHYSICAL EDUCATION MAJORS. Bambed, 32(2):84-90.

[6] Hermes-Lima, M.; Minuz, K.C. and Coutinho, I.S. (2002) THE RELEVANCE OF STUDENTS SEMINARS ON CLINICALLY RELATED SUBJECTS IN A BIOCHEMINSTRY COURSE FOR MEDICAL AND NUTRITION STUDENTS. Bambed, 30(1):30-434.

[7] Bianco, A.A.G. and Torres, B.B. (2004) A BIOCHEMISTRY DISCIPLINE DESIGNED FOR THE NUTRITION COURSE. Revista Brasileira de Ensino de Bioquímica e Biologia Molecular Http://sbbq.iq.usp.br/revista/artigo.php?artigoid $=98$

[8] Machado, M.J.; Rovaris, M.L. and Falkenberg, M. (2003) LEARNING MARKERS OF CARDIAC DAMAGE DISCIPLINE ON PHARMACY GRADUATE PROGRAM AT THE FEDERAL UNIVERSITY OF SANTA CATARINA (PPGFAR/UFSC): THE IMPACT OF BIOCHEMISTRY KNOWLEDGE BY STUDENTS PERCEPTION. Revista Brasileira de Ensino de Bioquímica e Biologia Molecular http://www.sbbq.org.br/revista/artigo.php?artigoid=85

[9] Preté, P.S.C.; Yokaichiya, D.K.; Molnar, A.M.; Kassab, B.H.; Covizi, D.Z.; Pando, L.A.; Gallão, M.I.; Pando, S.C.; Teixeira, S.A.; Malheiros, S.V.P.; Macedo, D.V.; De Paula, E. \& Torres, B.B. (1999) BIOQUÍMICA DA NUTRIÇÃO: UMA DISCIPLINA PLANEJADA E APLICADA POR PÓS-GRADUANDOS. XIV Reunião Anual da Federação de Sociedades de Biologia Experimental - FeSBE, Caxambu - MG. 
[10] Pinto, L.M.A.; Gonçalves, P.R.; Belline, P.; Granjeiro, P.A.; Messias Jr, N.S.; de Paula, E.; Macedo, D.V. and Torres. B.B. (1999) BIOCHEMISTRY OF WATER: A MULTIDISCIPLINAR APPROACH. XXVIII Reunião Anual da Sociedade Brasileira de Bioquímica e Biologia Molecular - SBBq, Caxambu - MG.

[11] Neto, A.P.C.; Oliveira, D.G.; Abreu, I.N.; Ribeiro, P.G.; Márquez, R.F.; de Paula, E.; Macedo, D.V.; Hoffmann, M.E. and Torres, B.B. (2000) BIOCHEMISTRY OF DRUGS: A DISCIPLINA FOR UNDERGRADUATE STUDENTS. XXIX Reunião Anual da Sociedade Brasileira de Bioquímica e Biologia Molecular - SBBq, Caxambu - MG.

[12] Trigoni Jr., R.; Carreira, A.C.; Correia, P.R.M.; Gonçalves, L.R.P.; Hatanaka, E.; Okuda, K.; Sá, P.L.Jr.; Silveira, R.V.M. and Torres, B.B. (2002) BIOCHEMISTRY OF CANCER: A DISCIPLINE FOR UNDERGRADUATES. Revista Brasileira de Ensino de Bioquímica http://www.sbbq.org.br/revista/artigo.php?artigoid $=47$

[13] Bianco, A.A.G.; Beton, D.; Carvalho , A.Z.1; Koide, T.; Miyamoto, S.; Ribichich, K.F.; Rodrigues, L.O.;Silva, F.H.L.; Tejada, E.C.S. and Torres, B. B. (2004) NUTRITION AND SPORTS: A BIOCHEMICAL APPROACH. Revista Brasileira de Ensino de Bioquímica e Biologia Molecular http://sbbq.iq.usp.br/revista/artigo.php?artigoid $=97$

[14] Yokaichiya, D.K.; Galembeck, E. and Torres, B.B. (2004) ADAPTING A BIOCHEMISTRY COURSE TO DISTANCE EDUCATION. Bambed, 32(1):27-29. 